\title{
The prevalence and risk factors of inhibitor development of FVIII in previously treated patients with hemophilia A
}

\author{
Ju Young Kim, Chur Woo You \\ Department of Pediatrics, Eulji University School of Medicine, Daejeon, Korea
}

p-ISSN 2287-979X / e-ISSN 2288-0011 https://doi.org/10.5045/br.2019.54.3.204 Blood Res 2019;54:204-209.

Received on November 21, 2018

Revised on March 5, 2019

Accepted on July 31, 2019

\section{Correspondence to}

Chur Woo You, M.D.

Department of Pediatrics, Eulji University School of Medicine, 95 Dunsanseo-ro,

Seo-gu, Daejeon 35233, Korea

E-mail: YCW1@eulji.ac.kr

(C) 2019 Korean Society of Hematology

\begin{abstract}
Background
Risk factors for the development of inhibitors in previously untreated patients (PUPs) have been reported; this is not the case in previously treated patients (PTPs) owing to fewer studies. Risk factors may differ for the development of PTP versus PUP inhibitors. We aimed to identify risk factors for PTP inhibitor development.

\section{Methods}

Participants were patients at a hemophilia treatment center in Korea with current or past history of factor VIII or factor IX alloantibodies. Observed inhibitors were classified as PUP or PTP inhibitors based on the cumulative number of exposure days. We compared the type and severity of hemophilia, mutation type, and family history of inhibitor between PUPs and PTPs. Events within 3 months before the first inhibitor detection, such as change of the factor concentrate used, short-term high exposure or continuous infusion of factor concentrate, history of surgery, infection, diagnosis of cancer, use of immunosuppressive or immunomodulator agents, and vaccination were compared between PUPs and PTPs.

Results

We observed 5 PUP inhibitors and 5 PTP inhibitors in 115 patients with hemophilia A. Events that might be related to the development of inhibitors within 3 months prior to the first inhibitor detection were observed in all 5 PTPs. On the contrary, no such events were observed in any PUPs. The observed events included a change in the factor concentrate used, subsequent chemotherapy, and short-term high exposure to factor concentrates for controlling hemorrhage and surgeries.
\end{abstract}

\section{Conclusion}

Our results suggest a greater role of nongenetic factors in PTP inhibitor development.

\section{INTRODUCTION}

Since it was first reported by Lawrence and Johnson in 1941, development of alloantibodies to factor VIII (FVIII) or factor IX (FIX) remains a great problem in the treatment of hemophilia [1]. This condition makes bleeding control difficult and hinders prophylactic treatment; therefore, more arthropathies and disabilities are observed in patients with such inhibitors. Furthermore, the cost of treatment becomes greatly increased mainly due to the high cost of using bypassing agents [2]. Inhibitors develop in approximately 30\% of patients with hemophilia A (HA) and 1-3\% of those with hemophilia B. Most inhibitors develop in patients with severe hemophilia who have mutations causing a CRM-negative state during the first (early) exposure days (EDs) to FVIII or FIX. However, inhibitors rarely develop after the first 150-200 EDs [3-5]. In reference to this observation, the International Society on Thrombosis and Haemostasis (ISTH) has defined inhibitors that develop after 150 EDs as previously treated patient (PTP) inhibitors [6].

In patients with $\mathrm{HA}$, previously untreated patient (PUP) inhibitors develop at a rate of $30 \%$ per patient-year. Many patient-oriented and non-patient-oriented risk factors that may be related to the development of inhibitors have been reported [1, 7-10]. On the contrary, PTP inhibitors in patients with HA develop at a rate of $0.1-0.6 \%$ per patient-year. Risk factors for the development of these inhibitors have 
not been elucidated owing to the rareness of these inhibitors and related studies [11, 12]. Risk factors associated with the development of PTP inhibitors may differ from those for PUP inhibitors. Presumably, more non-patient-oriented factors may exist in patients who already have immune tolerance to factor VIII (FVIII) during the first EDs. Currently, the number of elderly patients with hemophilia is increasing owing to the increased life expectancy of patients with hemophilia, which is comparable to the life expectancy of healthy patients without hemophilia in developed countries [13, 14]. Elderly patients with hemophilia have higher risk of exposure to non-patient-oriented factors such as infection, surgery, and cancer, which may break down their immune tolerance to FVIII or FIX, than in non-elderly patients with hemophilia, and PTP inhibitors may subsequently develop. Recent data in the United Kingdom (UK) support this possibility, showing a second peak of inhibitor development around the 60 s in patients with severe HA (SHA), most of whom were PTPs [15]. Therefore, it is important to elucidate the preventable risk factors related to the development of PTP inhibitors to prevent or at least decrease the incidence of these inhibitors. However, there are few studies that assess the risk factors in PTPs; consequently, the risk factors for the development of these inhibitors have not been elucidated. Therefore, this study aimed to identify risk factors for the development of inhibitors in PTPs.

\section{MATERIALS AND METHODS}

This study included patients at a hemophilia treatment center in Korea (Eulji University Hospital) who had a current record or history of alloantibodies to FVIII or FIX. Information on history of inhibitors was obtained from medical records of all patients with hemophilia registered in Eulji University Hospital from 2000 to 2018. An inhibitor titer above 0.6 Bethesda Units (BUs) was defined as positive. Only inhibitors with positive titers on two occasions were considered.

For each patient with an inhibitor, the cumulative number of EDs to FVIII/FIX concentrates before inhibitor development, the dates of detection and titers of the first two positive inhibitors, and the date of resolution of inhibitors were collected from medical records and patient diaries. The cumulative number of EDs was evaluated up to 200 EDs. When inhibitors were observed after 200 EDs, treatment years with clotting factor concentrates before inhibitor development were evaluated instead of EDs.

Each observed inhibitor was classified as a PUP or PTP inhibitor based on the cumulative number of EDs to FVIII/FIX concentrate before inhibitor development. We used the ISTH definitions to define PUP inhibitors as those that developed in a patient within 150 EDs and PTP inhibitors as those that developed after 150 EDs. Additionally, observed inhibitors were classified as high-responding and low-responding inhibitors depending on the inhibitor titer (5 BU) and degree of immune response to FVIII or FIX. Transient inhibitors were defined as inhibitors that disappeared within several months of continuous exposure to FVIII or FIX.

Clinical characteristics, such as type and severity of hemophilia, age at detection of inhibitor, mutation type, family history of inhibitor, and treatment type (prophylaxis vs. on-demand) during the previous 1 year before inhibitor development, were obtained from medical records of patients with inhibitors; these data were compared between PUPs and PTPs. To identify non-patient-related factors for inhibitor development, possible events within 3 months before the first inhibitor detection, such as change of the factor concentrate used, short-term high exposure to factor concentrate to prevent and control bleeding, and continuous infusion of factor concentrate, were obtained from medical records of patients with inhibitors. Additionally, we also obtained as much information as possible on the history of surgery, infection, diagnosis of cancer and/or treatment, use of immunosuppressive and/or immunomodulator agents, and vaccination within 3 months prior to the first inhibitor detection using the medical records of patients with inhibitors. These data were then compared between PUPs and PTPs.

Appropriate IRB approval was obtained for this study (IRB no. 2018-06-011).

\section{RESULTS}

Prevalence of inhibitors in patients with hemophilia

No inhibitors were observed in 14 patients with hemophilia B. On the contrary, 10 inhibitors were observed in 115 patients with HA (8.7\%); 7 inhibitors were high responding, 1 inhibitor was low responding, and the other 2 inhibitors

Table 1. Prevalence of inhibitors in patients with hemophilia.

\begin{tabular}{lcc} 
& Hemophilia A & Hemophilia B \\
\hline All Patients & $115(100 \%)$ & $14(100 \%)$ \\
Severe & $89(77.39 \%)$ & $5(35.7 \%)$ \\
Moderate & $21(18.26 \%)$ & $2(14.3 \%)$ \\
Mild & $5(4.3 \%)$ & $7(50 \%)$ \\
Inhibitor prevalence in All & & \\
Transient inhibitors included & $10 / 115(8.7 \%)$ & 0 \\
Transient inhibitors not included & $8 / 115(7.0 \%)$ & \\
Inhibitor prevalence in SH & & \\
Transient inhibitors included & $9 / 89(10.1 \%)$ & 0 \\
Transient inhibitors not included & $7 / 89(7.9 \%)$ & \\
$\quad$ High responding & $6(67 \%)$ & \\
$\quad$ Low responding & $1(11 \%)$ & \\
$\quad$ Transient inhibitor & $2(22 \%)$ & \\
Inhibitor prevalence in & $1 / 26(3.8 \%)$ & 0 \\
moderate and mild hemophilia & & \\
$\quad$ High responding & 1 & \\
Low responding & 0 & \\
Transient inhibitor & 0 & \\
\hline
\end{tabular}

Abbreviation: $\mathrm{SH}$, severe hemophilia. 
were transient inhibitors. Immune tolerance induction (ITI) was not given to any patients with inhibitors because ITI is not reimbursed; thus, except transient inhibitors, the other inhibitors persisted during the observation period. A total 9 of 10 inhibitors were observed in 89 patients with SHA (10.1\%). In contrast, only 1 (high-responding) inhibitor was observed in 26 patients with non-SHA (3.8\%) (Table 1).

\section{Prevalence rate and characteristics of inhibitors developed in} PTPs with hemophilia A

Of the 10 inhibitors observed in 115 patients with HA, 5 inhibitors developed within the first 150 EDs (PUP inhibitors; $4.3 \%, 5 / 115)$; the other 5 developed after 150 EDs (PTP inhibitors; 4.5\%, 5/110). All 5 PTP inhibitors developed in 85 patients with SHA (5.9\%, 5/85); 4 were high responding, and the fifth was a low-responding inhibitor. No transient inhibitors were observed in PTPs. The average age at inhibitor detection was 5.15 years (range, 1.6-11 yr) and 51.0 years (range, 33-70 yr) in PUPs and PTPs, respectively. The average number of EDs at inhibitor detection was 34.25 EDs (range, 20-50 EDs) in PUPs, and the average number of treatment years in PTPs was 32.6 years (Table 2).

\section{Risk factors for inhibitor development in PTPs with hemophilia A}

Events that might be related to the development of inhibitors within 3 months prior to the first inhibitor detection were observed in all 5 PTPs. On the contrary, no such events were observed in any PUPs. The observed events included a change in the factor concentrate used, one case of colon cancer surgery and subsequent chemotherapy, short-term

\begin{tabular}{|c|c|c|}
\hline & PUP inhibitor & PTP inhibitor \\
\hline \multicolumn{3}{|l|}{ Prevalence in SHA } \\
\hline TR included & 4/89 (4.5\%) & $5 / 85(5.9 \%)$ \\
\hline TR not included & $2 / 89(3.4 \%)$ & $5 / 85(5.9 \%)$ \\
\hline Prevalence in mod. HA & $0 / 21(0 \%)$ & $0 / 21(0 \%)$ \\
\hline Prevalence in mild HA & $1 / 5(20 \%)$ & $0 / 4(0 \%)$ \\
\hline \multicolumn{3}{|l|}{ All } \\
\hline TR included & $5 / 115(4.3 \%)$ & $5 / 110(4.5 \%)$ \\
\hline TR not included & 3/115 (2.6\%) & $5 / 110(4.5 \%)$ \\
\hline High response & $3(60 \%)$ & $4(80 \%)$ \\
\hline Low response & 0 & $1(20 \%)$ \\
\hline Transient response & $2(40 \%)$ & 0 \\
\hline $\begin{array}{l}\text { Average age of inhibitor } \\
\text { detection (yr) }\end{array}$ & $\begin{array}{l}5.15 \\
\quad \text { (range, 1.6-11 yr) }\end{array}$ & $\begin{array}{l}51.0 \\
\text { (range, 33-70 yr) }\end{array}$ \\
\hline $\begin{array}{l}\text { Average EDs of } \\
\text { inhibitor detection in } \\
\text { PUP/treatment years } \\
\text { in PTP }\end{array}$ & $\begin{array}{l}\text { 34.25 EDs } \\
\text { (range, 20-50 EDs) }\end{array}$ & $\begin{array}{l}32.6 \text { treatment } \mathrm{yr} \\
\text { (range, } 11-45 \mathrm{yr} \text { ) }\end{array}$ \\
\hline
\end{tabular}

Abbreviations: EDs, exposure days; HA, hemophilia A; PTP, previously treated patient; PUP, previously untreated patient; SHA, severe hemophilia $A ; T R$, transient inhibitor. high exposure to factor concentrates for controlling intra-abdominal hemorrhage and emergency splenectomy caused by a car accident, and orthopedic surgery (a knee arthroplasty and a right ankle osteotomy and synovectomy) (Table 3). Three PTP inhibitors, all high-responding inhibitors, were observed in three siblings with SHA who had been treated on-demand. Inhibitor developed at age 55 years in one brother after short-term high exposure to factor concentrates for the control of intra-abdominal hemorrhage and emergency splenectomy after a car accident, another brother developed inhibitor at age 65 years after colon cancer surgery and chemotherapy. A third brother developed inhibitor at 70 years old after right knee arthroplasty. The observed mutation among these family members was the Arg1997Trp missense mutation, which has been reported as a mutation in moderate and SHA. Other mutations observed in PTPs were one frame shift mutation with small deletions and one inversion 22 in each patient. The mutations observed in PUPs were one multi-exon deletion, two stop mutations, one inversion 22 mutation in patients with SHA, and a missense mutation observed in a patient with mild HA (Table 3).

\section{DISCUSSION}

The prevalence of inhibitors observed in patients with HA in this study was comparable to that in a meta-analysis of prevalence data for inhibitors developed in patients with HA [3-5]. In our study, the prevalence of inhibitors, including transient inhibitors, was $8.7 \%$ (10/115) in 115 patients with HA and $10.1 \%$ in 89 patients with SHA; this was $7.0 \%$ and $7.9 \%$ in all patients with HA and SHA, respectively, when transient inhibitors were excluded. Nearly all inhibitors developed in SHA, and only one inhibitor, a high-responding inhibitor in a patient with mild HA, was observed among the 26 patients with non-SHA. The prevalence in non-severe patients with HA was $3.8 \%(1 / 26)$ (Table 1$)$.

It is known that most factor of VIII/IX inhibitors arise after relatively few EDs to factor VIII, early in the patient's life in severe and moderate hemophilia; after 150-200 EDs, the subsequent risk of inhibitor development is very low [3-7]. Based on this observation, the ISTH has differentiated inhibitors as PUP inhibitors and PTP inhibitors [6].

PTP inhibitors rarely develop in patients with HA (0.1$0.6 \%$ per patient-year) [7]. Thus, it had been believed that most inhibitors are observed in PUPs. On the contrary, in our study, the cumulative number of inhibitors was the same for PUPs and PTPs (5 inhibitors each), and the prevalence of PUPs and PTPs was not different: $4.3 \%$ in PUPs and $4.5 \%$ in PTPs. Furthermore, when excluding two transient inhibitors observed only in PUPs, the prevalence was $2.6 \%$ and $4.5 \%$ in PUPs and PTPs, respectively (Table 2). Thus, more inhibitors of significance were observed in PTPs. A recent study on the incidence of inhibitors in patients with SHA in the UK, which analyzed prospective reports in the UK National Hemophilia Database of all new inhibitors reported between 1990 and 2009, showed that new inhibitors 
Table 3. Possible risk factors for inhibitor development in previously untreated patients and previously treated patients with hemophilia A.

\begin{tabular}{|c|c|c|c|c|c|c|c|c|c|c|c|}
\hline \multicolumn{6}{|c|}{ PTP inhibitor } & \multicolumn{6}{|c|}{ PUP inhibitor } \\
\hline $\begin{array}{l}\text { Case } \\
\text { No. }\end{array}$ & $\begin{array}{c}\text { Risk } \\
\text { factors }\end{array}$ & Dz type & $\begin{array}{l}\text { Inh. } \\
\text { type }\end{array}$ & Mutations & FMHX & $\begin{array}{l}\text { Case } \\
\text { No. }\end{array}$ & $\begin{array}{c}\text { Risk } \\
\text { factors }\end{array}$ & Dz type & $\begin{array}{l}\text { Inh. } \\
\text { type }\end{array}$ & Mutations & FMHX \\
\hline No. 1 & Exposure of new Ag & SHA & HR & $\begin{array}{l}1 \text { frame shift } \\
\text { mutation } \\
\text { with small } \\
\text { deletion }\end{array}$ & $(-)$ & No. 6 & $(-)$ & SHA & HR & $\begin{array}{l}\text { Multi-exon } \\
\text { deletion }\end{array}$ & $(-)$ \\
\hline No. 2 & $\begin{array}{l}\text { Cancer and surgery, } \\
\text { chemotherapy }\end{array}$ & SHA & HR & Arg1997Trp & $\begin{array}{l}(+) \text { Brother } \\
\text { of No. } 3 \text { and } \\
4 \text { patients }\end{array}$ & No. 7 & $(-)$ & SHA & $\mathrm{HR}$ & Stop & $(-)$ \\
\hline No. 3 & $\begin{array}{l}\text { Trauma and short-term } \\
\text { large exposure }\end{array}$ & SHA & HR & Arg1997Trp & $\begin{array}{l}\text { (+) Brother } \\
\text { of No. } 2 \text { and } \\
4 \text { patients }\end{array}$ & No. 8 & $(-)$ & SHA & TR & Stop & $(-)$ \\
\hline No. 4 & Orthopedic surgery & SHA & HR & Arg1997Trp & $\begin{array}{l}(+) \text { Brother } \\
\text { of No. } 2 \text { and } \\
3 \text { patients }\end{array}$ & No. 9 & $(-)$ & SHA & TR & Inv 22 & $(-)$ \\
\hline No. 5 & $\begin{array}{l}\text { Orthopedic surgery } \\
\text { and prophylaxis for } \\
\text { PT }\end{array}$ & SHA & LR & Inv22 & $(-)$ & No. 10 & $(-)$ & Mild HA & $\mathrm{HR}$ & Glu518Asp & $(-)$ \\
\hline
\end{tabular}

${ }^{a)}$ Events within 3 months before inhibitor detection.

No 1. Inhibitor detected at 32 years old and detected at 35 EDs after changing to EHL (extended half-life) FVIII.

No 2. Inhibitor detected at 65 years old after rectosigmoid cancer resection and chemotherapy.

No 3. Inhibitor detected at 55 years old after treatment for hemoperitoneum and liver laceration due to car accident, controlled with plasma-derived FVIII.

No 4. Inhibitor detected at 70 years old after knee arthroplasty.

No 5. Inhibitor detected at 33 years old after Rt with ankle osteotomy with synovectomy and prophylaxis for physical therapy.

Abbreviations: Dz, disease; EDs, exposure days; EHL, extended half-life; FMHX, familial history of inhibitor; HR, high responding; Ihn,

inhibitor; Inv, inversion; LR, low responding; PT, physical therapy; SH, severity of hemophilia; SHA, severe hemophilia A; TR, transient inhibitor.

may present throughout the life of patients with SHA, with a bimodal risk pattern that is greatest in early childhood $(<5 \mathrm{yr})$ and old age ( $>60 \mathrm{yr})[15]$. Nearly all inhibitors during this later peak are believed to be PTP inhibitors; inhibitors developed in patients with previously acquired tolerance to FVIII during their first EDs. An increasing frequency of PTP inhibitors among elderly patients with SHA, which is not reflected in earlier reports, may be emerging because patients are living longer [13, 14]. Although the ages during the later peak were lesser than those of the UK study, a similar trend was observed in patients with HA in this study, with an average age of inhibitor development of 5.15 (1.6-11) years and 51.0 (33-70) years in PUPs and PTPs, respectively, and no inhibitors developed in patients aged 11-33 years.

The mechanism for the development of inhibitors in PTPs is unknown, except in the case of new FVIII antigen exposure owing to a change in antigenic factor concentrates. The risk of new FVIII antigen exposure in PTPs was first witnessed in Austria and the Netherlands after exposure to new plasma-derived pasteurized FVIII concentrate (FVIII CPS-P) during 1990-1991 [16]. This was also observed after the introduction of recombinant factors in the early 2000s, although the incidence was very low in PTPs $(0-1.2 \%$ of the cohorts under investigation) $[11,12]$. In addition to the known risk of new antigen exposure, presumably, a breakdown of previous tolerance may be another mechanism for the development of inhibitors in PTPs because these patients have had many hundreds of FVIII EDs. This breakdown of tolerance may reflect circumstances (danger signals such as surgery and intensive replacement therapy) and/or deterioration in immune regulation with advancing age. Anecdotal evidence suggests that these inhibitors frequently arise after intense replacement therapy during surgery or to control bleeding ("peak treatment moments"), a risk factor that has been established in children with SHA and patients with mild HA $[17,18]$. Thus, nongenetic factors that would break down tolerance may contribute to the development of PTP inhibitors more than genetic factors. However, only a few studies on this issue have been conducted among PTPs; furthermore, nongenetic factors that would lead to the breakdown of tolerance were not included in most of these studies. Consequently, no risk factors, except the previously known risk of new antigen exposure, have been elucidated [11, 12].

In this study, anecdotal events that might be related to the development of inhibitors in PTPs were observed in all 5 PTPs with inhibitors. On the contrary, no such events were observed in any of the 5 PUPs with inhibitors. The observed events were a change in factor concentrate, colon cancer surgery and chemotherapy, trauma due to a car accident and subsequent short-term high exposure to factor concentrate, and orthopedic surgery (Table 3). Except for one case of new antigen exposure, all the other events may have contributed to the breakdown of tolerance to FVIII. Although our study population included a small number of cases at 
a single center, these results suggest that nongenetic factors may contribute to the development of inhibitors to a greater degree in PTPs than in PUPs because patient-oriented factors, such as hemophilia severity and mutation types, were not different between these groups (Table 3).

Despite these findings, reflecting the anecdotal risk factors of inhibitor development in patients with mild and moderate HA [18], some patient-oriented factors, such as family history of inhibitor and certain high-risk mutations, may presumably contribute to the development of PTP inhibitor. For example, 3 PTP inhibitors, all high-responding inhibitors, were observed in three brothers with SHA in this study. They had been treated on-demand for years, and inhibitors developed over age 55 years after events such as surgery, chemotherapy, and trauma with subsequent short-term high exposure to factor concentrate. The observed mutation among these family members was the Arg1997Trp missense mutation, which has been reported in moderate and SHA [19]. This finding suggests that there may be certain high-risk F8 gene mutations and/or immunologic genetic factors in patients who are prone to developing PTP inhibitors. This may lead a patient with HA to break down tolerance easier than other patients, when nongenetic risk factors are superimposed on genetic risks, with subsequent PTP inhibitor development. Arg1997Trp may be such a high-risk mutation for the development of PTP inhibitor in patients with HA. However, this mutation has not been reported as a high-risk mutation, and neither type of inhibitor has been previously reported in patients with this mutation [19]. Therefore, this should be confirmed in studies including more patients who have this missense mutation.

The present study was conducted in a relatively small number of patients at a single center in Korea. Thus, it is necessary to conduct national study in Korea to confirm the bimodal risk of inhibitor development during a patient's life, especially for the later peak of inhibitors, most of which developed in PTPs. Although our study findings suggest a greater role of nongenetic factors in inhibitor development among PTPs, a national study would serve to confirm these findings.

\section{Authors' Disclosures of Potential Conflicts of Interest}

No potential conflicts of interest relevant to this article were reported.

\section{REFERENCES}

1. Oldenburg J, Pavlova A. Genetic risk factors for inhibitors to factors VIII and IX. Haemophilia 2006;12:15-22.

2. Saint-Remy JM, Jacquemin MG. Inhibitors to factor VIII: immunology. In: Lee CA, Berntorp EE, Hoots K, eds. Textbook of hemophilia. Chichester, UK: John Wiley \& Sons, 2014:41-7.

3. Lusher JM, Arkin S, Abildgaard CF, Schwartz RS. Recombinant factor VIII for the treatment of previously untreated patients with hemophilia A. Safety, efficacy, and development of inhibitors. Kogenate Previously Untreated Patient Study Group. N Engl J Med 1993;328:453-9.

4. Rothschild C, Laurian Y, Satre EP, et al. French previously untreated patients with severe hemophilia A after exposure to recombinant factor VIII : incidence of inhibitor and evaluation of immune tolerance. Thromb Haemost 1998;80:779-83.

5. Yee TT, Williams MD, Hill FG, Lee CA, Pasi KJ. Absence of inhibitors in previously untreated patients with severe haemophilia A after exposure to a single intermediate purity factor VIII product. Thromb Haemost 1997;78:1027-9.

6. White GC, DiMichele D, Mertens K, et al. Utilization of previously treated patients (PTPs), noninfected patients (NIPs), and previously untreated patients (PUPs) in the evaluation of new factor VIII and factor IX concentrates. Recommendation of the Scientific Subcommittee on Factor VIII and Factor IX of the Scientific and Standardization Committee of the International Society on Thrombosis and Haemostasis. Thromb Haemost 1999;81:462.

7. Dimichele DM, Lacroix-Desmazes S, Peyvandi F, Srivastava A, Rosendaal FR; Subcommittee on Factor VIII, Factor IX and Rare Coagulation Disorders. Design of clinical trials for new products in hemophilia: communication from the SSC of the ISTH. J Thromb Haemost 2015;13:876-9.

8. Goodeve A. The incidence of inhibitor development according to specific mutations and treatment? Blood Coagul Fibrinolysis 2003;14:S17-21.

9. Viel KR, Ameri A, Abshire TC, et al. Inhibitors of factor VIII in black patients with hemophilia. N Engl J Med 2009;360:1618-27.

10. Santagostino E, Mancuso ME, Rocino A, et al. Environmental risk factors for inhibitor development in children with haemophilia A: a case-control study. Br J Haematol 2005;130:422-7.

11. Kempton CL, Soucie JM, Abshire TC. Incidence of inhibitors in a cohort of 838 males with hemophilia A previously treated with factor VIII concentrates. J Thromb Haemost 2006;4:2576-81.

12. Xi M, Makris M, Marcucci M, Santagostino E, Mannucci PM, Iorio A. Inhibitor development in previously treated hemophilia A patients: a systematic review, meta-analysis, and meta-regression. J Thromb Haemost 2013;11:1655-62.

13. Sultan Y. Prevalence of inhibitors in a population of 3435 hemophilia patients in France. French Hemophilia Study Group. Thromb Haemost 1992;67:600-2.

14. Darby SC, Kan SW, Spooner RJ, et al. Mortality rates, life expectancy, and causes of death in people with hemophilia A or $B$ in the United Kingdom who were not infected with HIV. Blood 2007;110:815-25.

15. Hay CR, Palmer B, Chalmers E, et al. Incidence of factor VIII inhibitors throughout life in severe hemophilia A in the United Kingdom. Blood 2011;117:6367-70.

16. Rosendaal FR, Nieuwenhuis HK, van den Berg HM, et al. A sudden increase in factor VIII inhibitor development in multitransfused hemophilia A patients in The Netherlands. Dutch Hemophilia Study Group. Blood 1993;81:2180-6.

17. Gouw SC, van der Bom JG, Marijke van den Berg $H$. Treatment-related risk factors of inhibitor development in previously untreated patients with hemophilia A: the CANAL cohort study. Blood 2007;109:4648-54. 
18. Hay CR, Ludlam CA, Colvin BT, et al. Factor VIII inhibitors in mild and moderate-severity haemophilia A. UK Haemophilia Centre Directors Organisation. Thromb Haemost 1998;79:762-6.
19. You CW, Son HS, Kim HJ, Woo EJ, Kim SA, Baik HW. Mutation analysis of factor VIII in Korean patients with severe hemophilia A. Int J Hematol 2010;91:784-91. 